\title{
Características de la educación sexual escolar recibida y su asociación con la edad de inicio sexual y uso de anticon- ceptivos en adolescentes chilenas sexualmente activas
}

\author{
Electra González A. MSc ${ }^{1 a}$, Temístocles Molina G. ${ }^{1 b}$, Carolina Luttges D. ${ }^{1 c}$ \\ ${ }^{1}$ Centro de Medicina Reproductiva y Desarrollo Integral del Adolescente, Facultad de Medicina, Universidad de Chile. \\ ${ }^{a}$ Asistente Social. ${ }^{b}$ Bioestadístico. ${ }^{\mathrm{C}}$ Psicóloga.
}

\section{RESUMEN}

Antecedentes: La educación sexual integral en el contexto escolar es uno de los pilares fundamentales para prevenir embarazos e infecciones de transmisión sexual en adolescentes. Objetivo: Analizar las características de la educación sexual escolar recibida y su asociación con la edad de inicio sexual y uso de anticonceptivo en adolescentes consultantes en un centro de salud sexual y reproductiva. Método: Estudio analítico de corte transversal realizado en 1.232 adolescentes mujeres de nivel socioeconómico medio y bajo atendidas en un centro de salud sexual y reproductiva durante 2007-2011. La información fue obtenida desde una entrevista estructurada aplicada al ingreso. Resultados: El 92,8\% reportó haber recibido alguna forma de educación sexual en el colegio, un $51,6 \%$ reconoció el colegio como principal fuente de información en métodos anticonceptivos. Tener como fuente principal de conocimiento en métodos anticonceptivos el colegio fue asociado a inicio sexual más tardío y uso de método anticonceptivo. Conclusión: Los resultados del estudio muestran que dada la prevalencia de conocimientos anticonceptivos $(92,8 \%)$ y que solo el $1 \%$ de las adolescentes que había recibido educación sexual en sus respectivos colegios se había embarazado podemos afirmar que la educación sexual recibida por las adolescentes del estudio fue al menos adecuada.

PALABRAS CLAVE: Educación sexual, anticoncepción, inicio sexual, adolescentes

\section{SUMMARY}

Background: Comprehensive sex education in the school context is one of fundamental pillars to prevent pregnancy and sexually transmitted infections in adolescents. Aim: to analyze the characteristics of the sexuality information received in school and its association with age of sexual initiation and contraceptive use in adolescents consulting a center for sexual and reproductive health. Methods: Analytical crosssectional study performed in 1,232 adolescent women of medium and low socio-economic status attending to a sexual and reproductive health center during 2007-2011. Information was obtained from a structured interview applied at first visit. Results: $92.8 \%$ reported receiving some form of sex education at school, $51.6 \%$ recognized the school as the main source of information on contraception. Having as a main source of knowledge in school contraceptives was associated with later sexual initiation and contraceptive use. Conclusion: The study results show that given the contraceptive prevalence $(92.8 \%)$ knowledge and that only $1 \%$ of adolescents who had received sex education in their schools had been pregnant can say that sex education received by adolescents study was at least adequate.

KEY WORDS: Sex education, contraception, sex initiation, adolescents 


\section{INTRODUCCIÓN}

Reducir el embarazo adolescente y las infecciones de transmisión sexual (ITS) incluido el VIH/ SIDA son metas de políticas públicas en nuestro país pero hasta ahora las estrategias han sido insuficientes y poco efectivas (1).

En Chile, como en muchos países desarrollados, los y las adolescentes están iniciando actividad sexual a edades más tempranas que en generaciones anteriores. La evidencia científica muestra que el inicio de la actividad sexual a más temprana edad puede tener consecuencias psicológicas, sociales y económicas negativas $(2,3)$. Por otro lado, el inicio sexual temprano expone a los y las adolescentes a tener comportamientos sexuales de riesgo, entre ellos, múltiples parejas sexuales (que pueden ser secuenciales, a lo largo de la vida, o que éstas sean concurrentes o simultáneas), o a ser inconsistentes en sus prácticas sexuales más seguras (uso de anticonceptivos). La combinación de estos factores los y las ponen en riesgo de una variedad de problemas que incluyen los embarazos no planeados y la adquisición de infecciones de transmisión sexual (3-7).

Para promover actitudes y comportamientos más saludables es fundamental la educación en sexualidad escolar, la cual busca entregar a niños, niñas y personas jóvenes, el conocimiento, las competencias y los valores que les permitan asumir responsabilidad sobre su vida sexual y social. Una educación en sexualidad planteada desde un enfoque integral, con énfasis en prevención del embarazo adolescente, ITS, VIH/SIDA, está relacionada con un comienzo más tardío de la actividad sexual, un menor número de parejas sexuales y un uso más amplio y sistemático del preservativo. Además, contribuye a mejorar las actitudes hacia las personas que viven con VIH y puede reducir el estigma y la discriminación (8-10).

En países desarrollados, principalmente europeos, como Suiza y los países nórdicos que tienen una larga historia de educación sexual en el sistema educacional, demuestran una tendencia sostenida de baja tasas de embarazo adolescente y bajas tasas de ITS en adolescentes, reconociendo que hay otros factores sociales, familiares, culturales y de servicios de salud que pueden influir en sus tasas de fecundidad $(11,12)$.

Sin embargo, introducir programas de educación sexual en el sistema escolar en países más conservadores como es el caso de Chile, produce muchas resistencias debido a que los detractores argumentan que el sexo y la sexualidad son asuntos privados que no deben ser discutidos en público y menos con los niños pequeños $(13,14,15)$.

A pesar que está probado que el temprano acceso a la información en sexualidad es la mejor estrategia, no solo de impedir las ITS, embarazos no planeados, abortos inseguros, violencia sexual, entre otros, en nuestro país la educación en sexualidad en los colegios ha sido hasta ahora un proceso inadecuado, fragmentado, mínimo y tardío (16-20).

En 1993 se formula una Política de Educación Sexual en Chile pero no fue explícitamente obligatoria dentro del ordenamiento jurídico chileno. La implementación de los programas quedó a merced de la libertad de enseñanza de cada escuela.

En los años 1990 y 1996 se implementaron las Jornadas de Conversación sobre Afectividad y Sexualidad, JOCAS. Apoyada técnica y financieramente por UNFPA, que tuvo un gran impacto a nivel cultural, social y mediático. A pesar de las dificultades y resistencia por los sectores más conservadores, las JOCAS se mantuvieron hasta el 2000. Su cobertura había superado los 600 liceos en el país.

En 2005 el Ministerio de Educación creó una comisión especial para implementar el Plan de Educación en Sexualidad y Afectividad 2005-2010 el cual consideró el tema de Afectividad y Sexualidad dentro de los Objetivos Fundamentales Transversales a nivel del Marco Curricular Obligatorio. Estos objetivos ya habían sido insertos tanto en la Educación Básica en 1996, como en la Educación Media en 1998, y posteriormente en las Bases Curriculares para la Educación Parvularia en 2002. Sin embargo, el cumplimiento de estos objetivos no tuvo el impacto esperado ni la cobertura del plan anterior $(20,21)$. En 2010 recién se implementó por ley la obligatoriedad de la Educación Sexual Escolar, aunque solo para la enseñanza media. Es importante destacar que el cumplimiento de la Ley 20.418, compromete tanto al Ministerio de Educación como al de Salud en materia de educación sexual (22).

Aun cuando esta información en sexualidad escolar haya sido fragmentada, mínima y tardía, es posible que algún impacto tenga en las actitudes y comportamientos sexuales en las adolescentes de nuestro país. Este conocimiento puede ser esencial para promover con mayor fuerza la instalación de programas de educación sexual escolar integrales, adecuados y tempranos.

El objetivo de este estudio fue analizar las características de la educación sexual escolar recibida y su asociación con la edad de inicio de la actividad sexual y el uso de anticoncepción en mujeres adolescentes consultantes en un centro de salud sexual y reproductiva universitario.

\section{MATERIAL Y MÉTODO}

Diseño de estudio transversal analítico, que analizó las características de la educación en sexualidad recibida y su asociación con la edad de inicio de la actividad sexual y con el uso de métodos anticonceptivos, previo al ingreso al Centro 
de Medicina Reproductiva y Desarrollo Integral del Adolescente (CEMERA). La población del estudio fueron mujeres adolescentes entre 12 y 19 años de la Región Metropolitana que consultaron en CEMERA durante el período 2007-2011. CEMERA es el primer y más importante centro de atención integral en salud sexual y reproductiva para adolescentes en el país, situado en la capital de Chile, que tiene como principal objetivo posponer el primer embarazo. El estudio obtuvo la información de la entrevista estructurada, diseñada para propósitos de investigación que aplicó la investigadora principal al ingreso de cada adolescente y que fueron ingresados a una base de datos. A todas las adolescentes se les explicó la modalidad de atención y se les solicitó permiso explícito para utilizar los antecedentes que quedaron registrados en la entrevista para fines de investigación, asegurando el resguardo de la confidencialidad y anonimato de los datos. Lo anterior fue formalizado mediante firma del consentimiento informado especialmente diseñado para estos efectos.

Durante el periodo 2007 a 2011, 3.814 adolescentes consultaron en el CEMERA. Fueron incluidas en este estudio aquellas adolescentes que reportaron haber tenido información en sexualidad en el colegio y habían iniciado actividad sexual coital. Cumplidos los criterios de elegibilidad la población final fue de 1.232 adolescentes.

Las variables sociodemográficas estudiadas fueron: edad al momento de la entrevista, actividad (estudia, no estudia), escolaridad (curso actual), rendimiento escolar (medido por promedio de notas del último curso aprobado), aspiraciones académicas futuras, nivel socio-económico (usando la escala modificada de Graffar adaptada a Chile) (23).

Las variables relacionadas con las características de información en sexualidad analizadas fueron: con quien conversa sobre sexualidad, información en sexualidad en la niñez recibida en el hogar (si/no), cuáles fueron las principales fuentes de información en MAC, información de sexualidad en el colegio (si/no), curso en que recibió la primera

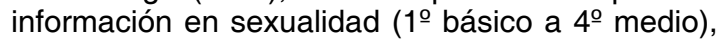
primer tema de sexualidad recibido. Nivel de conocimiento de métodos anticonceptivos (bueno, regular, malo). Nivel de conocimiento de métodos de prevención de ITS (bueno, regular, malo). Las variables dependientes fueron: edad de inicio sexual y uso de método anticonceptivo previo al ingreso al CEMERA (si/no).

El plan de análisis consistió primero en identificar las variables sociodemográficas y de comportamiento de la población en estudio; luego relacionar las características de la información en sexualidad recibida y edad de inicio sexual como las características de la información en sexualidad recibida y uso previo de método anticonceptivo.
Análisis estadístico. Para evaluar la asociación entre las variables de interés y el uso previo de método anticonceptivo se aplicó la prueba estadística de Chi cuadrado. Para evaluar las diferencias de medias entre las variables de interés y la variable edad de inicio sexual se utilizaron las siguientes pruebas estadísticas: T de Student, Test de MannWhitney, Análisis de Varianza (ANOVA) con post hoc Scheffe y Kruskal-Wallis. Para los análisis estadísticos se utilizó el software STATA 10.0.

El estudio fue aprobado por el Comité de Ética en Seres Humanos de la Facultad de Medicina de la Universidad de Chile. La confidencialidad y manejo de la información fue resguardada rigurosamente.

\section{RESULTADOS}

Los resultados corresponden a 1.232 adolescentes mujeres entre 12 y 19 años. La edad promedio a la entrevista fue de 15,7 años, con un promedio de escolaridad de 10 años, el rendimiento escolar alcanzó a un 5,5 en la escala de 1 a 7 . El $64,5 \%$ tenía como aspiraciones académicas seguir estudios superiores. En cuanto al nivel socioeconómico, el $5,3 \%$ pertenece al nivel alto, el $57,2 \%$ al nivel medio y $37,5 \%$ al nivel bajo. Las adolescentes eran estudiantes de colegios municipalizados o particular subvencionado de la Región Metropolitana.

El promedio de edad de inicio de la actividad sexual fue de 15 años. El 50,5\% reporta haber usado algún tipo de anticoncepción previo ingreso al CEMERA. El 29,4\% conversa temas de sexualidad solo con amigos/as, 34\% lo hace con la madre o el padre. El $97 \%$ refiere conocer la mayoría de los métodos anticonceptivos y el $84,9 \%$ conoce el condón como método más efectivo para la prevención de ITS. El 92,8\% de las adolescentes reportan que recibieron información en sexualidad en el colegio mientras que casi el $48 \%$ la recibió también en la familia. En relación a la principal fuente de información sobre métodos anticonceptivos, un $51,6 \%$ reporta que fue el colegio y un $18 \%$ los padres (Tabla I).

El $35,8 \%$ de las adolescentes reporta que el $7^{\circ}$ básico fue el curso en que recibieron los primeros temas en sexualidad, le sigue el $8^{\circ}$ básico con un $22,4 \%$ y $6^{\circ}$ básico con un $12,9 \%$ (Figura 1 ). El $43 \%$ de las adolescentes reporta que el primer tema abordado en el colegio fue sobre prevención de embarazo e infecciones de transmisión sexual, el $37,9 \%$ señala que fueron aspectos biológicos y de reproducción, un $9,7 \%$ afectividad y pololeo y $9,4 \%$ indica otros (Tabla I). En $7^{\circ}$ y $8^{\circ}$ básico el tema que más se aborda es prevención de embarazo e infecciones de transmisión sexual (Figura 2). 
Tabla I

CARACTERÍSTICAS SOCIO-DEMOGRÁFICAS DE LAS PARTICIPANTES DEL ESTUDIO

Variables
Motivo de ingreso
Uso de anticonceptivos al inicio de la actividad sexual

Con quien conversa sobre sexualidad

Conocimiento sobre métodos anticonceptivos

Conocimiento prevención de infecciones de transmisión sexual (ITS)

Fuente principal de conocimiento de métodos anticonceptivos

Recibió información sobre sexualidad en casa

Recibió información sobre sexualidad en el colegio

Primeros temas recibidos

\begin{tabular}{lcc} 
Categorías & $\mathrm{n}$ & $\%$ \\
\hline Anticoncepción & 1019 & 82,7 \\
Embarazo & 12 & 1,0 \\
Ginecología & 138 & 11,2 \\
Medicina adolescente & 36 & 2,9 \\
Salud mental & 27 & 2,2
\end{tabular}

No

$595 \quad 49,5$

$\mathrm{Si}$

$608 \quad 50,5$

$\begin{array}{lcc}\text { Madre/padre } & 396 & 34,0 \\ \text { Familiares } & 272 & 23,4 \\ \text { Amigos/as } & 342 & 29,4 \\ \text { Pololo } & 61 & 5,2 \\ \text { Otro } & 93 & 8,0\end{array}$

Todos los métodos anticonceptivos $\quad 1163 \quad 97,0$ Solo anticonceptivos orales $14 \quad 1,2$

Solo condón $\quad 10 \quad 0,8$

$\begin{array}{lll}\text { No conoce } & 12 & 1,0\end{array}$

Uso condón

Pareja conocida

No conoce

1012

84,9

$11 \quad 0,9$

$169 \quad 14,2$

Colegio

$596 \quad 51,6$

Padres

Amigos

Medios de comunicación

Familiares

Profesionales de salud/otro

$207 \quad 17,9$

$133 \quad 11,5$

$101 \quad 8,7$

$53 \quad 4,6$

$66 \quad 5,7$

$\mathrm{Si}$

539

47,8

No

588

52,2

Si

1043

92,8

No

81

7,2

Prevención embarazo/anticoncepción

Aspectos biológicos y reproductivos

Otros

7

9,4




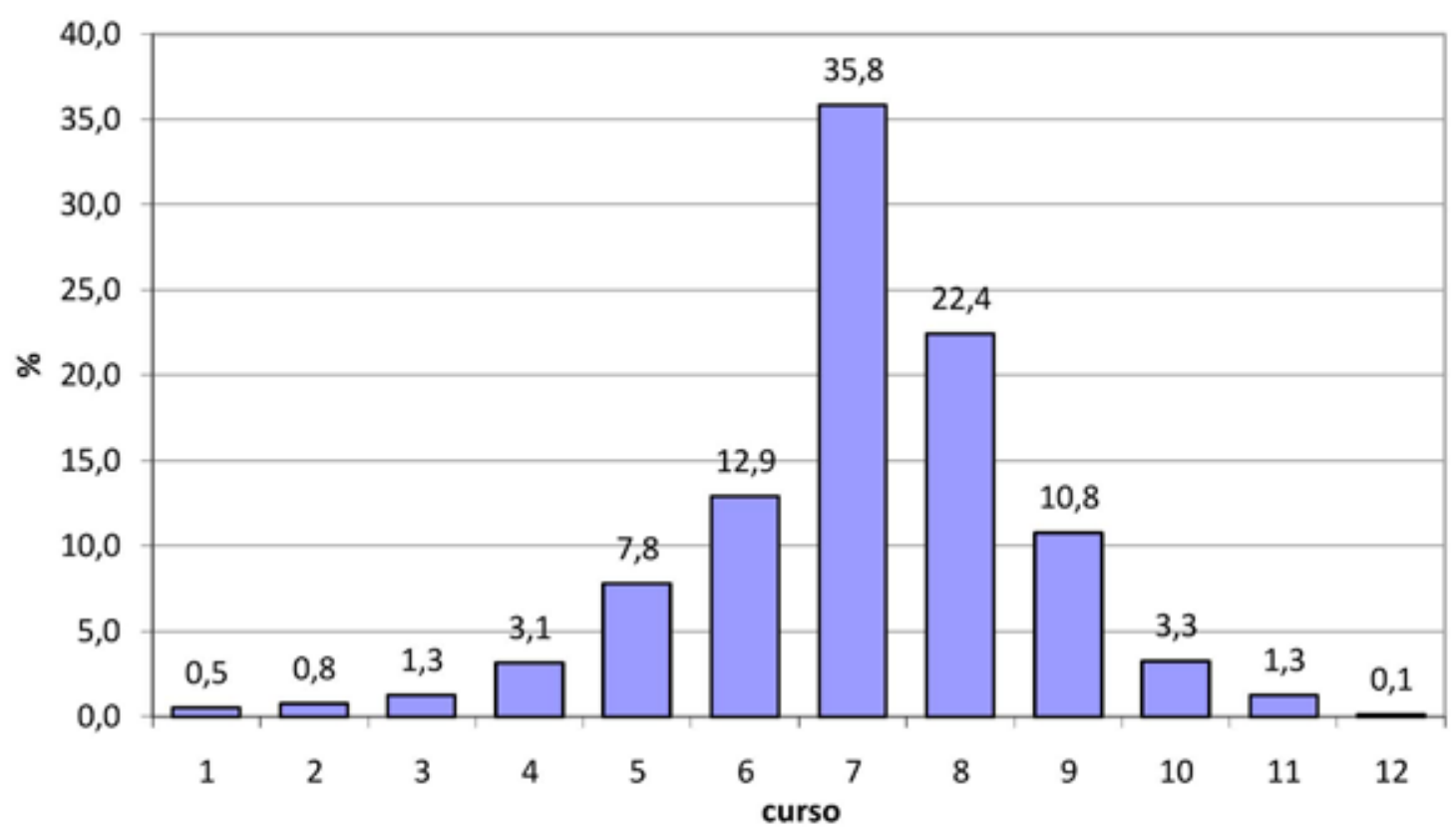

Figura 1. Distribución \% del curso en que recibe información en sexualidad en el colegio.

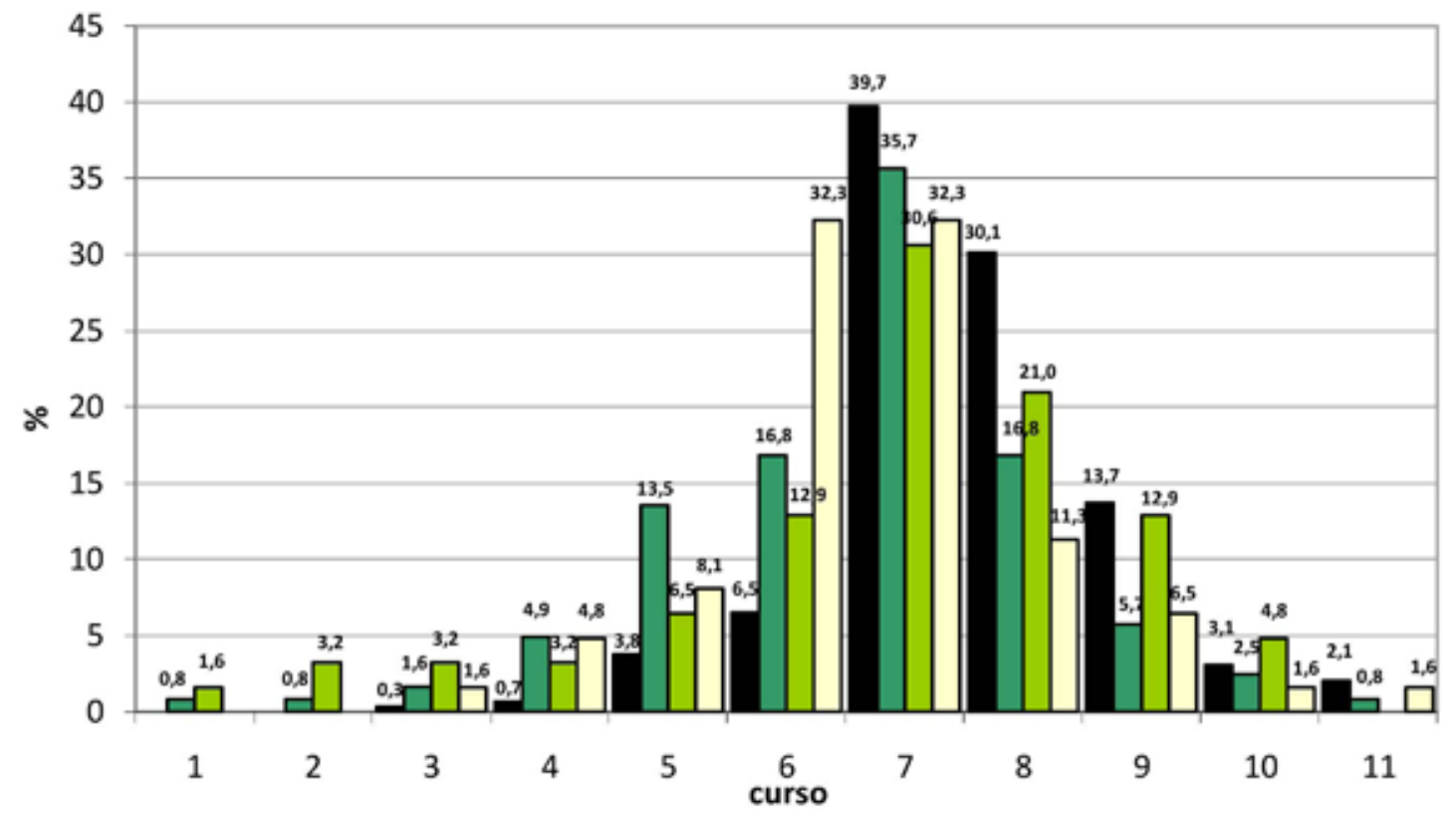

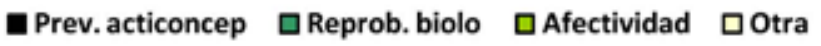

Figura 2. Distribución \% de los temas tratados en Educación Sexual por curso en que la reciben 
Al cruzar tiempo transcurrido entre la información en sexualidad recibida en el colegio y la edad de inicio de la actividad sexual se puede observar que el $15,9 \%$ de las adolescentes recibió esta información al momento o posterior al inicio de la actividad sexual (Figura 3). En las menores de 15 años, este porcentaje correspondió a un $25,3 \%$ mientras en las mayores de 15 años fue un $14,7 \%$, diferencia estadísticamente significativa $(p=0,00)$ (Figura 4). No hubo diferencia al cruzar por nivel socioeconómico. Al analizar a las adolescentes con uso previo de métodos anticonceptivos (MAC), el 13,6\% había recibido información sexual escolar tardía, mientras en que en las que no usaron MAC previo, esta cifra correspondió a 20,8\% ( $p=0,00)$ (Figura 5).

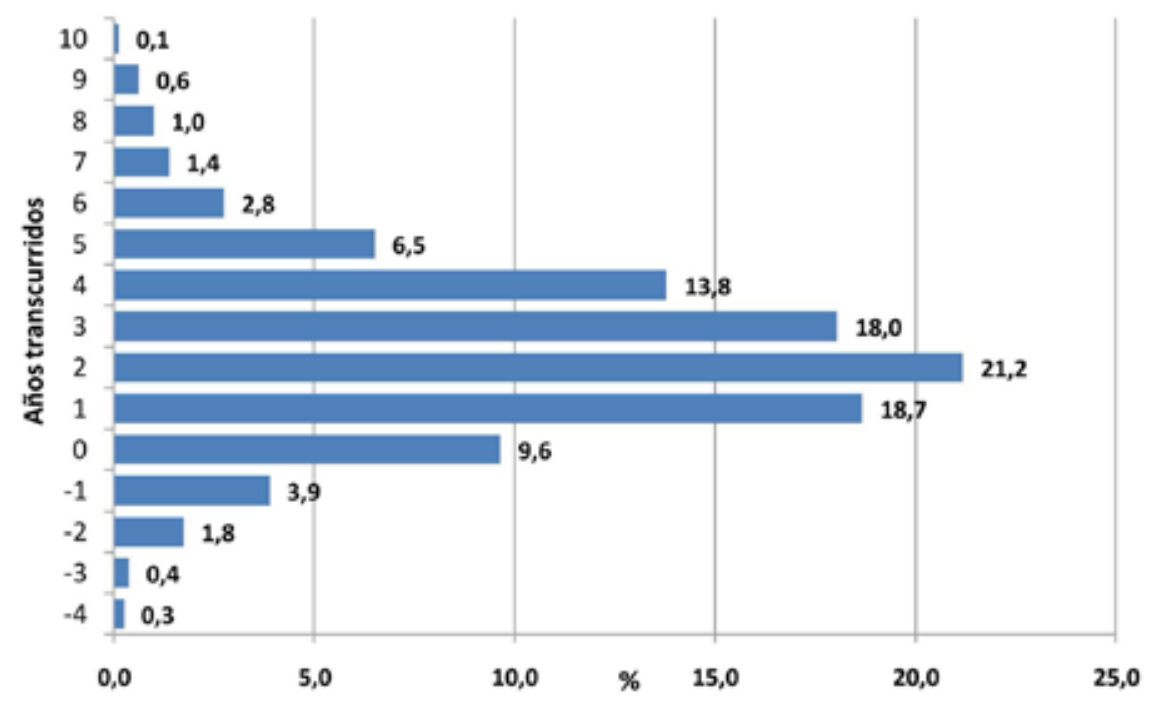

Figura 3. Frecuencia (\%) de años transcurridos entre la información en sexualidad recibida e inicio de la actividad sexual.

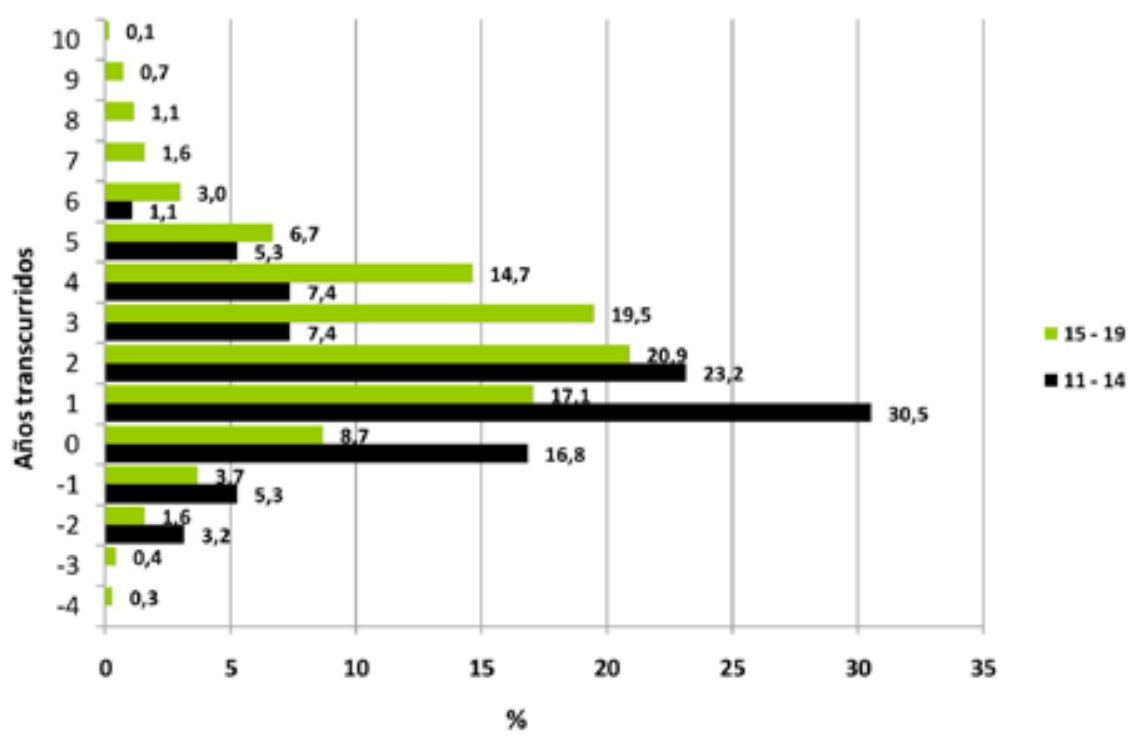

Figura 4. Frecuencia (\%) de años transcurridos entre información en sexualidad recibida e inicio de actividad sexual por grupo de edad. 


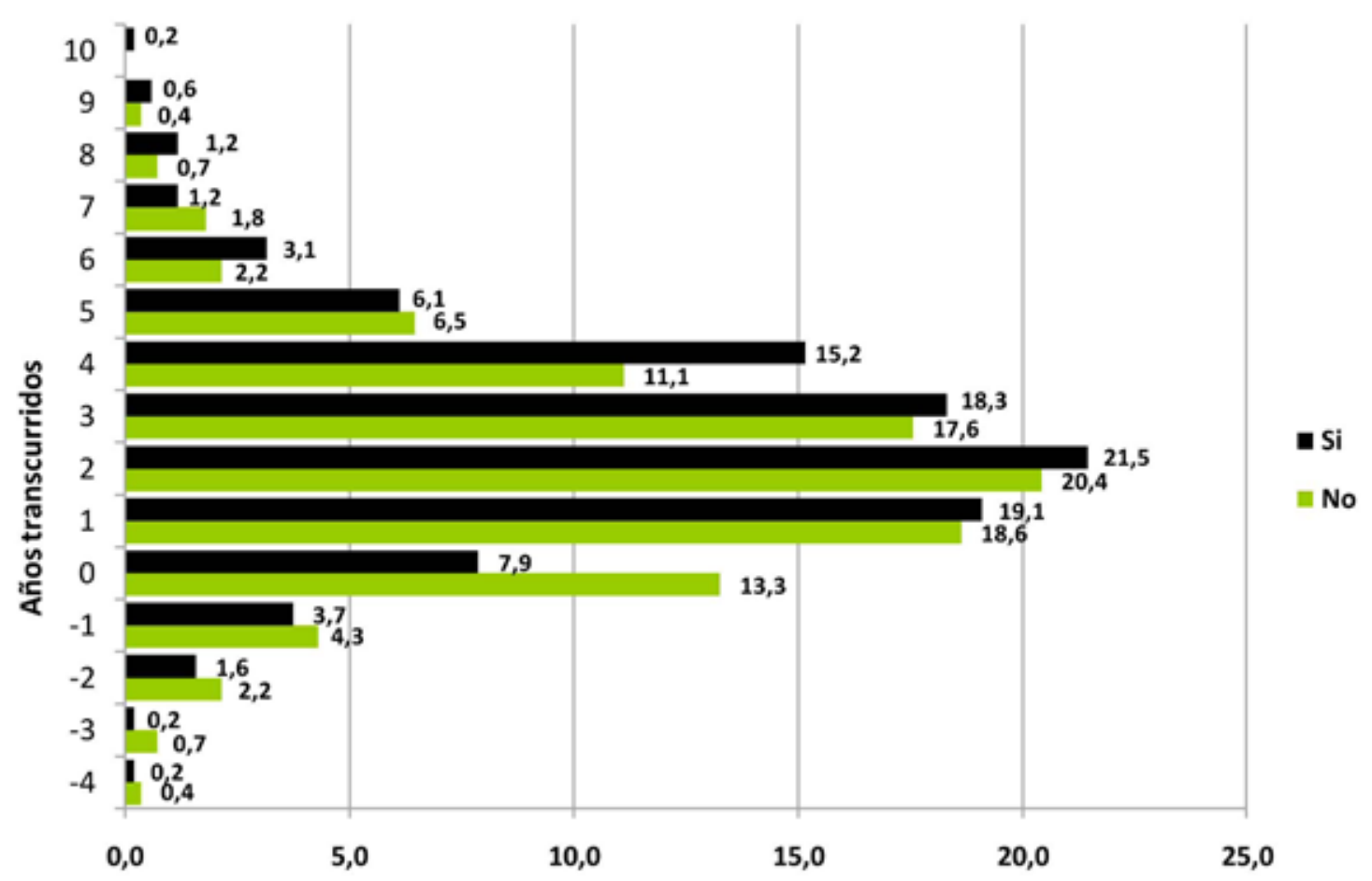

Figura 5. Frecuencia (\%) de años transcurridos entre información en sexualidad recibida e inicio sexual según uso o no uso de anticonceptivo al inicio de la actividad sexual.

El análisis bivariado muestra que las variables asociadas a menor edad de inicio de la actividad sexual fueron no ser estudiantes, tener como aspiraciones educacionales solo terminar enseñanza media. Mientras que, las variables asociadas al inicio sexual más tardío fueron: conversar de sexualidad con otros, reconocer el condón como método de prevención de ITS, tener el colegio como principal fuente de información sobre métodos anticonceptivos.

No mostró diferencias en el inicio sexual, nivel socioeconómico, nivel de conocimiento de MAC, tener información sobre sexualidad en la familia, tener información sexual en el colegio, el curso en que se impartió el primer tema de sexualidad y los temas abordados en el colegio (no mostrado en Tabla).

Las variables asociadas al uso de MAC previo fueron, haber recibido información de sexualidad en el colegio, reportar un buen nivel de conocimiento de MAC, haber recibido información sexual en enseñanza media y mayor edad. El resto de las variables estudiadas no mostró diferencia en el uso o no uso de MAC al inicio de la actividad sexual (no mostrado en Tabla).

\section{DISCUSIÓN}

Los resultados de nuestra investigación permiten establecer que la educación sexual recibida por adolescentes en sus centros educacionales ha permitido que el $92,8 \%$ tenga algún conocimiento sobre sexualidad y que el $51,6 \%$ reconoce que el colegio ha sido su principal fuente de información sobre métodos anticonceptivos. Tener como fuente principal de conocimientos en métodos anticonceptivos el colegio fue asociado al inicio sexual más tardío y a uso de método anticonceptivo inmediatamente al inicio de la actividad sexual. Estos resultados son relevantes dado que la educación sexual escolar junto con servicios de salud amigables y accesibles son dos estrategias fundamentales en la prevención del embarazo adolescente e infecciones de transmisión sexual, especialmente VIH$\operatorname{SIDA}(8,9,10)$.

De acuerdo a la información reportada por las entrevistadas, la educación sexual recibida no fue sistemática ni sostenida en el tiempo ya que los temas principalmente fueron impartidos en la asignatura de Ciencias Naturales y Biología en $7^{\circ}$ básico, y después en Orientación en $2^{\circ}$ medio. 
Por otro lado, si bien dos tercios de ellas reportan haber recibido los primeros temas entre $6^{\circ} \mathrm{y}$ $8^{\circ}$ básico, es decir cuando ellas tenían entre 12 y 14 años y cuando cambios del desarrollo puberal tan importantes como la menarquia, por ejemplo, ya habían ocurrido, esta fue recibida a tiempo si consideramos que el promedio de edad de inicio de la actividad sexual fue a los 15 años para esta población. Es importante además destacar que si bien la ley 20.418 solo obliga a impartir educación sexual a contar del primer ciclo de enseñanza media, observamos que la mayoría de los colegios la impartió entre $6^{\circ}$ y $8^{\circ}$ básico, es decir, más temprano de lo que la ley obliga.

El énfasis en los primeros contenidos recibidos en el colegio fue la prevención de embarazo (anticoncepción) y de ITS lo cual se asoció a inicio sexual más tardío y a uso de anticonceptivos en la población estudiada lo que fue concordante con lo reportado en la literatura $(8,9,10)$. Es decir, aun cuando la educación sexual escolar recibida pudo haber sido fragmentada y no sistemática, mostró asociación con los comportamientos sexuales de las adolescentes estudiadas.

Las limitaciones del estudio fue la imposibilidad de establecer la calidad y la extensión de la educación recibida, por la diversidad en las formas en que ésta fue impartida, (clases, charlas, talleres), en diferentes asignaturas y por quienes la impartieron (profesores del establecimiento escolar, profesionales de salud u por otros externos al colegio).

Por otro lado, considerando que el inicio sexual es un fenómeno multifactorial, el presente estudio solo se centró en la educación sexual recibida en el colegio. Los resultados son aplicables a adolescentes mujeres de la Región Metropolitana que solicitaron atención en salud sexual y reproductiva en un centro especializado, no pueden ser generalizados a la población adolescente de ambos sexos ni de otras regiones.

Sin embargo, a pesar de estas limitaciones, nuestros hallazgos son relevantes ya que las participantes son estudiantes de colegios municipalizados o particular subvencionado, por lo que corresponde a una población especialmente vulnerable y en necesidad de intervenciones destinadas a prevenir el inicio sexual temprano y aunque sabemos que los comportamientos sexuales son multifactoriales, la educación sexual escolar es una de las estrategias fundamentales para promover comportamientos sexuales más saludables como inicio sexual más tardío, menos parejas sexuales y un uso más efectivo y sistemático de métodos anticonceptivos efectivos $(16,17)$.

\section{CONCLUSIÓN}

Dada la prevalencia de conocimientos anticonceptivos $(92,8 \%$ ) y que solo el $1 \%$ de las adolescentes que había recibido educación sexual en sus respectivos colegios se había embarazado, podemos afirmar que la educación sexual recibida por las adolescentes del estudio fue al menos adecuada.

\section{REFERENCIAS}

1. De Bruyn M. Jóvenes en riesgo: Los adolescentes y la salud sexual, Agenda Salud, 2001;23:1-8. Isis Internacional, Santiago, Chile

2. González E, Molina T, Montero A, Martínez V. Comportamientos sexuales y diferencias de género en adolescentes usuarios de un sistema público de salud universitario. Rev Med Chile 2007;135:1261-69.

3. O'Donnell L, Donnell R C, Stueve A. Early sexual initiation and subsequent sex-related risks among urban minority youth: The Reach for Health Study. Fam Plann Perspect 2001;33:268-75.

4. Hofferth S. Factors affecting initiation of sexual intercourse. In: Risking the future: Adolescent sexuality, pregnancy and childbearing, Washington, DC: National Academy Press 1987;1:7-35.

5. SERNAM. Análisis de las conductas de las/os adolescentes Región Metropolitana, 1997, No. 53. Santiago, Chile,

6. Molina R, Molina T, González E. Madres niñas-adolescentes de 14 años y menos: Un grave problema de salud pública no resuelto en Chile. Rev Med Chile 2007;135:79-86.

7. González E, Molina T, Montero A, Martínez V, Leyton C. Factores asociados a la continuidad del uso anticonceptivo en adolescentes solteras nulíparas. Rev Med Chile 2009;137:1187-92.

8. Fernández L, Bustos M, González L, Palma D, Villagrán J, Muñoz S. Creencias, actitudes y conocimientos en educación sexual. Rev Med Chile 2000;128:574-83.

9. Gascón JA, Navarro B, Gascón FJ, Pérula de Torres LA, Jurado A, Montes G. Sexualidad y fuentes de información en población escolar adolescente. Med Fam 2003;4:124-9

10. Barbón Pérez O. Fuentes de información sobre educación sexual en adolescentes estudiantes de enfermería. Rev Cub Higiene Epid 2001;49:238-46.

11. Narring F, Michaud PA, Sharma V. Demographic and behavioural factors associate with adolescent pregnancy in Switzerland. Fam Plann Perspect 1996;28:232-6.

12. Berthoud R, Robson K. The outcomes of teenage motherhood in Europe, Innocenti Working Paper $n^{\circ} 86$, 2001, UNICEF Innocenti Research Centre, Florence, Italy.

13. Romero RJ, Lora MN, Cañete R. Adolescentes y fuentes de información de sexualidad: preferencias y utilidad percibida. Aten Primaria 2001;27:12-7

14. Phillips K, Martinez A. Sexual and reproductive health education: contrasting teachers health partners and former students perspectives. Can $\mathrm{J}$ Public Health 2010;101:374-9. 
15. Ruiz-Canela M, López del Burgo C, Carlos S, Calatrava M, Osorio A, Irala J. Familia, amigos y otras fuentes de información asociadas al inicio de las relaciones sexuales en adolescentes de EI Salvador. Rev Panam Salud Pública 2012;31:54-61.

16. Serrano I, Lete I, Coll C, Rabat A, Bermejo R, Martínez J, Doval JL, Pérez-Campos E, Dueñas JL. Anticonceptivos y fuentes de información sobre sexualidad y anticoncepción en la juventud española: resultados de una encuesta nacional. Rev Iberoam Fert Rep Hum 2012;29:28-34.

17. Kirby D. Abstinence, sex and STD/HIV education programs for teens: their impact on sexual behavior, pregnancy and sexually transmitted disease. Ann Rev Sex Res 2007;18:143-77.

18. Kim C, Free C. Recent Evaluations of the peer-led approach in adolescent sexual health education: a systematic review. Perspect Sex Reprod Health 2008;40:144-51.
19. Haghund A K, Fehring J R. The association of religiosity, sexual education and parental factors with risky sexual behaviors among adolescents and young adults. J Relig Health 2010;49:460-72.

20. Gaspar M, Reis M, Ramiro L, Borile M, Berner E, Vásquez S, González E, et al. Educação sexual em Portugal e em varios paises da America Latina. Psic Saúde \& Doenças 2009;10:149-58.

21. González E. Educación en sexualidad, afectividad desde una perspectiva de género. Paris $E$, Sánchez I, Beltramino D, Copto A. Meneghello (ed). En: Pediatría,6aㅡ ed. Tomo I, Buenos Aires, Editorial Médica Panamericana 2013; cap 36, pp 293-7

22. Ley 20.418, Diario Oficial del 28 Enero 2010.

23. Álvarez M, Muzzo S, Ivanovic D. Escala para medición del nivel socioeconómico en el área de salud. Rev Med Chile 1985;113:243-9. 